Case study

\title{
TUBERCULOSIS OF ORAL MUCOSA - A CASE REPORT
}

\author{
Emekar SM', Damle AS², Iravane J² \\ ${ }^{1}$ Dept. of Microbiology, PDVVPF Medical College, Viladghat, Ahmednagar (Maharashtra), India \\ 2 Dept. of Microbiology, Govt. Medical College \& Hospital, Aurangabad (Maharashtra),
}

\begin{abstract}
Tuberculosis (TB) is a chronic granulomatous disease and a leading cause of death in developing countries. Mucous membrane (oral/genital/perianal) TB could be either primary or secondary to autoinoculation of Mycobacterium tuberculosis from underlying advanced TB infection of lung, genitourinary or gastrointestinal tract respectively. Involvement of oral cavity in TB is quite rare, so difficult to get diagnosed. Hence we are reporting a case of TB of oral (buccal) mucosa in a 22 year old male who presented with painful ulcerative growth on left side of buccal mucosa near the molar teeth since 5 months. Diagnosis was made on histopathological examination and Ziehl Neelsen (ZN) staining of biopsy specimen. The complete blood count, chest X-ray, culture of tissue biopsy and sputum showed findings consistent with Mycobacterium tuberculosis infection. Patient responded to the treatment with complete recovery of tuberculous oral lesion. Hence awareness of rare form of oral tuberculosis makes early diagnosis and prevents further spread of disease.
\end{abstract}

Key words: Oral Tuberculosis, Langhans Cell.

\section{INTRODUCTION}

Tuberculosis has been the most important of human infections, in its global prevalence, devastating morbidity and massive mortality. ${ }^{1}$ South-East Asia carries a disproportionate 88 percent of the world's burden of TB. India accounts for nearly one-third of the global burden of TB.,3 The incidence of tuberculosis in the oral cavity is quite rare. It could be either primary or secondary to pulmonary disease. ${ }^{4}$ There are about $1-1.5 \%$ cases of pulmonary tuberculosis with associated oral cavity lesions. The oral sites most frequently affected are the tongue, palate, tonsil, pharynx, and buccal mucosa. ${ }^{5}$. The vulnerability to TB in developing

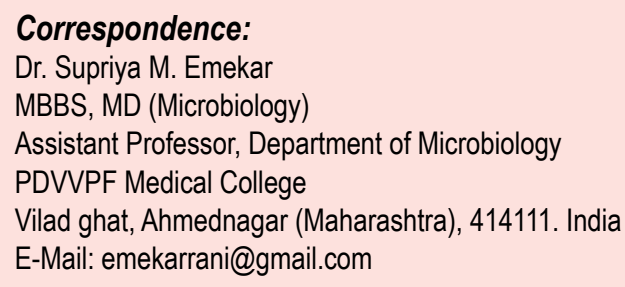

countries results from poverty, economic recession and malnutrition. ${ }^{2,3}$

The epidemic of HIV infection and development of multi drug resistant bacteria also contributes to increase in number of tuberculosis cases. With increasing number of tuberculosis cases, unusual forms of the disease are also likely to increase.

Oral lesions have a non-specific presentation varying from ulcers to granulomas, fissures and may be overlooked. ${ }^{4,6,7}$ Here we report a case of TB of oral (buccal) mucosa in $22 \mathrm{yr}$ old male.

\section{Case Report}

A 22 year old male presented to the Government Dental College and Hospital Aurangabad (Maharashtra) India, with painful ulcerative swelling on left side buccal mucosa near $2^{\text {nd }}$ molar tooth. The patient also had productive cough, weakness, weight loss and difficulty in opening mouth. These symptoms were gradually progressed over a period of five months. He was treated in private clinic symptomatically during this period but 
responded poorly. He had history of pulmonary TB one year back for which he had taken complete anti tuberculosis therapy (ATT) with Isoniazid 600 $\mathrm{mg}$, Rifampicin $450 \mathrm{mg}$ and Pyrazinamide 1500 $\mathrm{mg}$, Ethambutol $1200 \mathrm{mg}$ for two months followed by Rifampicin and Isoniazid for four months. He had no history of dental trauma or any oral surgery or tobacco chewing.

The oral ulcer was initially small and enlarged gradually over a period of five months. Oral examination revealed tender, shallow ulcer of size $1 \times 1.5 \mathrm{~cm}$ with irregular margins, covered with mucopurulent discharge and slough in the left retro molar trigone (figure. 1). On palpation ulcer was indurated and with undermined margins. Local examination showed diffuse swelling of left side of face. Cervical lymph nodes were neither enlarged nor fixed to surrounding tissue. Counseling was done and informed consent was obtained. Complete blood count (CBC) was normal. Erythrocyte sedimentation rate (ESR) was raised. Chest X-ray revealed widespread fibrocaseous infiltrate. The HIV antibody test was negative.

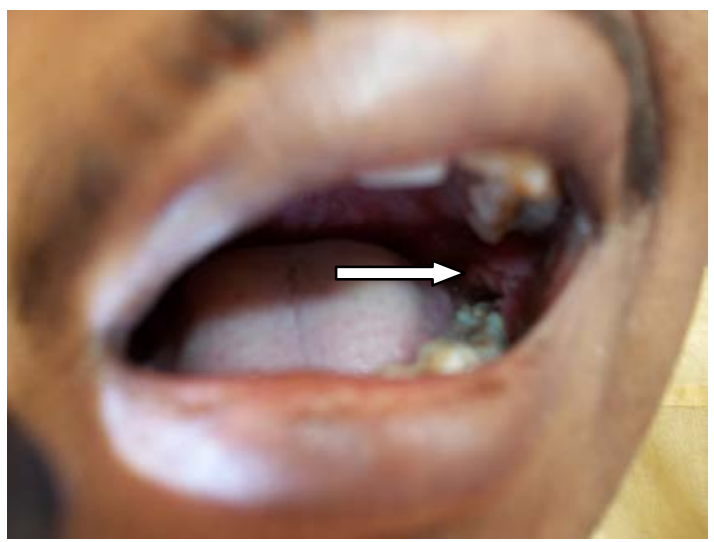

Figure 1. Oral ulcerative growth in left retro molar trigone

The patient was referred to the Microbiology and Pathology laboratory for confirmatory diagnosis. A biopsy was obtained from the margin of the lesion. Biopsy specimen was cut into tissue sections which were mounted on two glass slides. Histopathological examination with hematoxylin and eosin stain ( $\mathrm{H}$ and $\mathrm{E}$ stain) of the one tissue section showed lymphocytes, Langhans type giant cells and caseous necrosis. (figure.2). Another tissue section slide stained with ZN staining showed presence of acid fast bacilli within granuloma. A culture on Lowenstein and Jensen (LJ) media from sputum and tissue lesion grew characteristic dry,

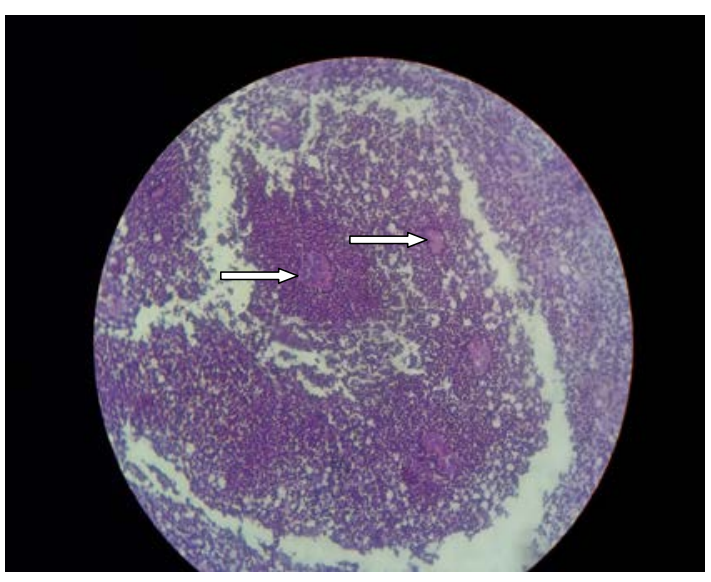

Figure 2. Histopathology ( $H$ and $E$ stain 10x) of buccal mucosal biopsy section showing 1. Langhans type giant cell and 2. foci of caesous necrosis and plenty of lymphocytes

rough, raised and irregular colonies after 30 days. It was confirmed as Mycobacterium tuberculosis on ZN staining and other biochemical tests.

The patient was confirmed as a case of oral (buccal) mucosal TB with the recurrence of pulmonary TB. ATT (category II regimen) was given after consultation with TB medical officer. Category II regimen consist of Isoniazid $600 \mathrm{mg}$, Rifampicin $450 \mathrm{mg}$, Pyrizinamide $1500 \mathrm{mg}$, Ethambutol 1200 $\mathrm{mg}$ and Streptomycin $750 \mathrm{mg}$ for two months then Isoniazide, Rifampicin, Pyrizinamide and Ethambutol for one month in intensive phase. This was followed by Isoniazide, Rifampicine, Ethambutol for five months. Patient's oral tuberculous lesion as well as general symptoms improved within three months.

\section{DISCUSSION}

Incidence of oral tubercular infections is quite rare, occurring in $0.05 \%-5 \%$ of all tuberculosis cases. ${ }^{9}$. In our case, buccal mucosa is involved.

A break in the epithelial continuity facilitates inoculation by the bacilli present in sputum. Both local and systemic predisposing factors exist for the occurrence of oral lesions. ${ }^{6}$. Local factors include poor oral hygiene, local trauma, presence of preexisting lesions such as leukoplakia, periapical granulomas, cysts, abscesses and periodontitis. ${ }^{4,6}$.

Systemic predisposing factors include primary or secondary immune suppression and nutritional deficiencies. 
Orificial tuberculosis usually results from autoinoculation of the infectious agent in patients with advanced internal tuberculosis of the lungs, gastrointestinal or genitourinary tract. Haematogenous or lymphatic dissemination from another active source of tuberculosis has also been described. ${ }^{9}$

Most of the TB cases occurring in the oral cavity are secondary to pulmonary infections, though other primary lesions are not unknown. ${ }^{4}$ Clinicians should also search and investigate for symptoms associated with primary TB (active source for secondary TB) which act as a source for orificial involvement.

Aird $^{10}$ has described five pathological types of oral tuberculous lesion as ulcers, tuberculoma, fissure, papilloma and cold abscess.

Shallow ulcerative growth with undermined edges was the presentation in our case.

The histopathology and culture of biopsy specimen was done for confirmation of oral (buccal) mucosal TB. The mucosal involvement was secondary to pulmonary TB.

Health care workers and the contacts of patients are at risk due to aerosol transmission. There is delay in diagnosis due to rarity of the condition. So clinician should be aware of mucosal TB and do the further investigations for favourable outcome in such cases.

\section{CONCLUSION}

A major concern about the cases of TB is the risk of transmission of tuberculosis in the community. Clinicians, especially dentists, ENT surgeons and general surgeons are involved in diagnosis and treatment of tuberculosis in rare presentation of oral mucosal TB. They are also exposed to the TB bacilli as occupational health hazard. Therefore it should be considered as differential diagnosis in chronic mucosal lesion in developing country like India. Clinicians' awareness will help in early diagnosis and prevent complications.

\section{Acknowledgement}

We are thankful to Professors in Department of Pathology and Department of Dentistry, Government Medical College, Aurangabad (Maharashtra), India for their kind cooperation, assistance and help to complete the study.

\section{REFERENCES}

1. R Anantnarayan, C K Jayaram Paniker. Textbook of Microbiology, $8^{\text {th }}$ edition, 2009, Universities Press (India) Private Limited.

2. Chadha VK. Epidemiological situation of tuberculosis in India. Indian $J$ Med Res. 2003;101:144-7.

3. Chakraborty AK. Epidemiology of tuberculosis; Current status in India. Indian $\mathrm{J}$ Med Res. 2004;120:248-76.

4. Rinaggio J. Tuberculosis. Dent Clin North Am. 2003;47:449-65.

5. Sharma $A B$, Laishram DK, Sarma B. Primary tuberculosis of tongue. Ind $\mathrm{J}$ Pathol Microbiol. 2008;51:65-6.

6. Prabhu SR, Sengupta SK: Bacterial infections due to mycobacteria, In Prabhu SR, Wilson DF, Daftary DK, Johnson NW, editors oral Diseases in the Tropics, (1st ed), Delhi Oxford university press, 1993;195-202,

7. Yepes JF, Sullivan J, Pinto A: Tuberculosis: medical management update oral Surg Oral Med Oral Pathol Oral Radiol Endod, 2004;267-73,

8. Mignogna MD, Muzio LLO, Favia G, Ruoppo E, Sammartino G, Zarrelli $C$ et al . Oral tuberculosis: a clinical evaluation of 42 cases oral Dis, 2000;2530 ,

9. Sehgal VN, Wagh SA. Cutaneous tuberculosis. Current concepts. Int J Dermatol 1990;29:237-52.

10. Khatri BK, Jhalla GS. Tuberculosis of tongue: a case report. Indian J Tuberc. 1971;18:58-9. 